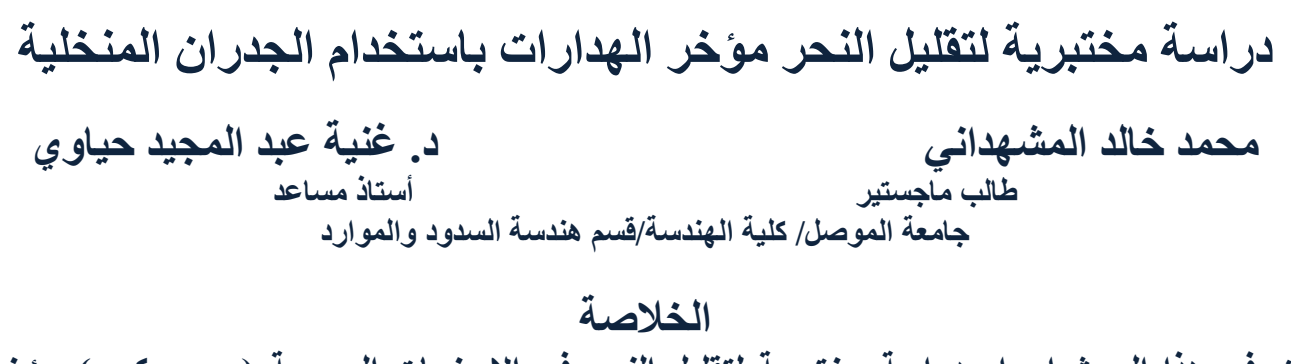

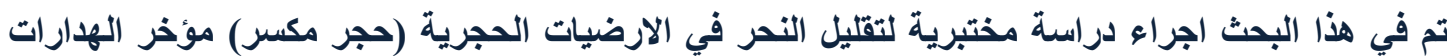

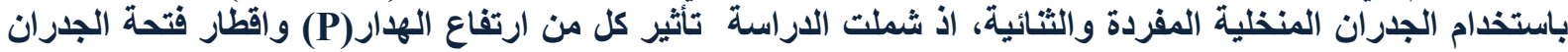

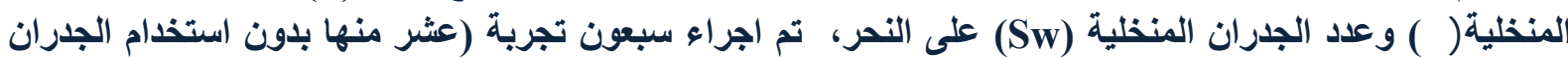

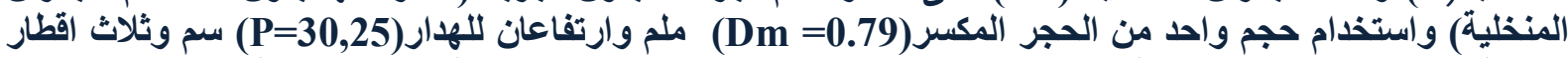

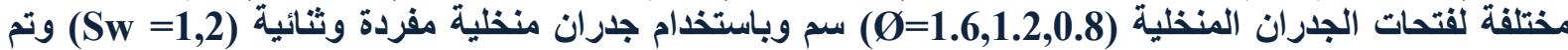

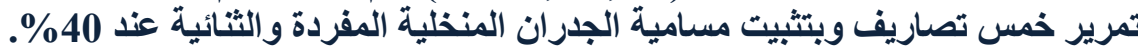

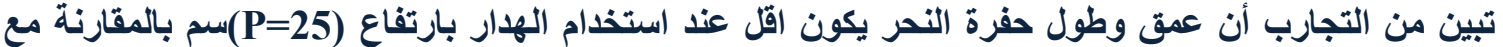

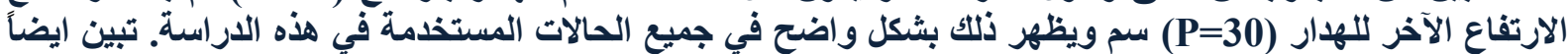

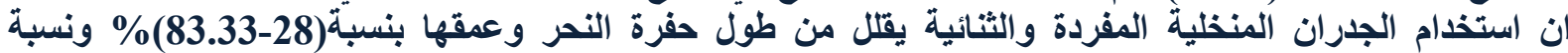

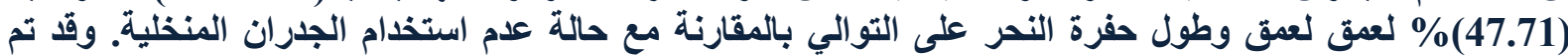

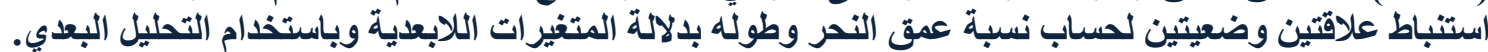

\title{
Laboratory Study to Decrease the Scour Downstream Weirs Using screen Walls
}

\begin{abstract}
In this research, a laboratory study of the phenomenon of scour in the stone floors (gravel, crushed) using single and double screen walls used as energy dissipation. The study includes the effect of weir height (P), the diameters of screen wall hole $(\varnothing)$, the number of screen walls ( $\mathrm{Sw}$ ) on scour behind walls screen. Seventy experiments were carried out (ten of them without using screen walls). One size of crushed stone (Dm $=$ 0.79) $\mathrm{mm}$ were used with two height of weir $(P=25,30) \mathrm{cm}$ and three different diameters of screen walls $(\varnothing=0.8,1.2,1.6) \mathrm{cm}$ were used and single and double screen walls $(\mathrm{Sw}=1,2)$ with five different discharge, with porosity $40 \%$ for single and double screen walls.

The results of the experimental work showed that the depth and length of the scour hole was minimum when using screen walls of $(P=25) \mathrm{cm}$ in comparison with $(P=30)$ $\mathrm{cm}$. Also the use of single and double screen walls decrease the depth and length of scour hole with ratios of $(28-83.33) \%$ and ratio of $(47.37-85.71) \%$ respectively compared with the state of without using screen. Using dimensional analysis two empirical relations were determined to compute the ratio of depth and length scour in term of dimensionless variables.
\end{abstract}

Keyword: Screen walls, Weirs Scour 


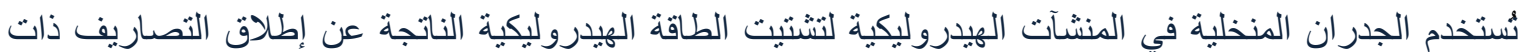

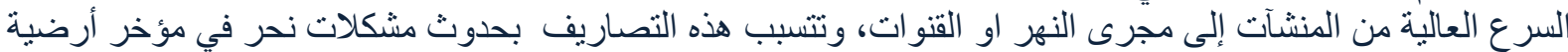

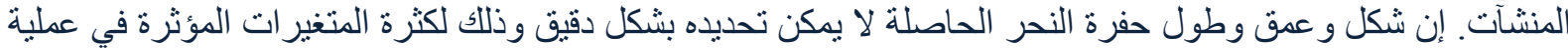
النحر هذه، و لأهية الموضوع أجريت دراسات وبحوث عديدة نظرية وتطبيقية لتحديد أبعاد مشكلات النحر الحاصلّة أسفل

المنشآت الهيدروليكية.

تشير كثير من الدراسات إلى أن الجدران المنخلية (Screen or perforated - Walls) تستخدم لتبديد الطاقة

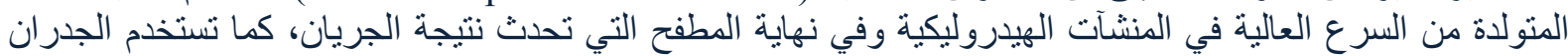

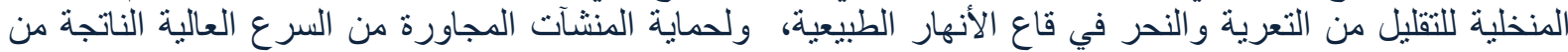

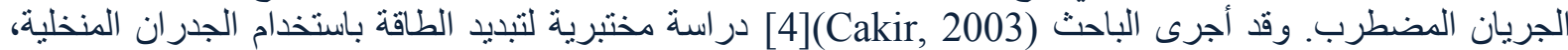
وكانت العو امل المتغيرة في التجارب هي المسامية وسمك وموقع الجدار ، والمسامية (

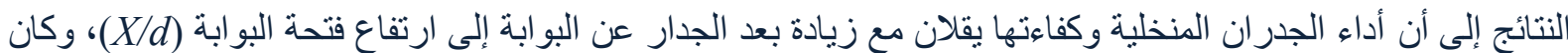

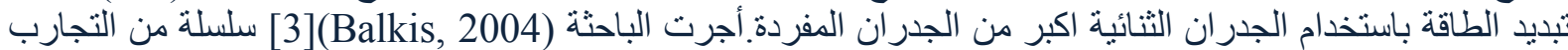

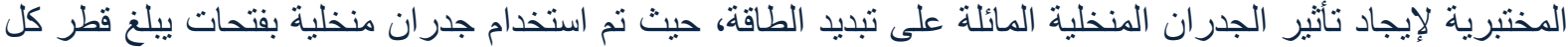

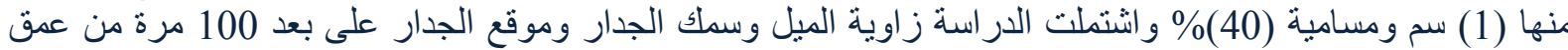

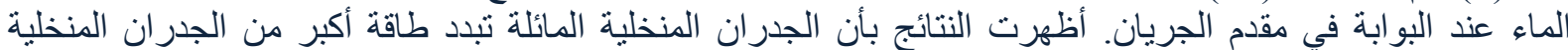

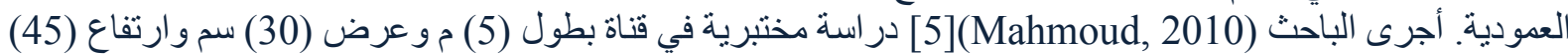

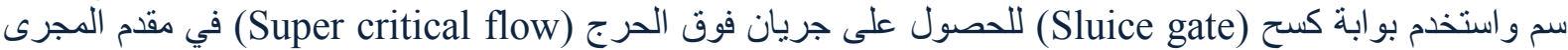
(upstream)

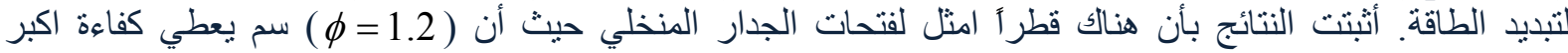

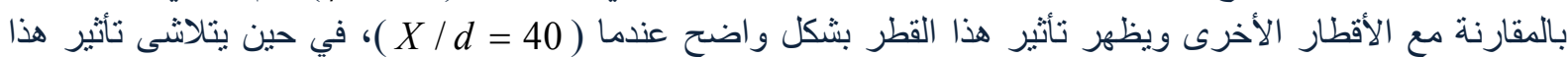

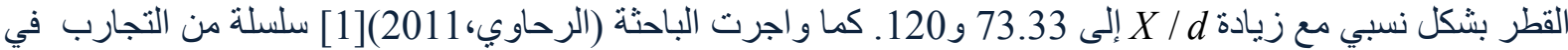

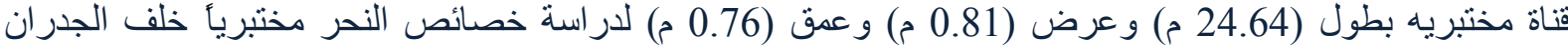

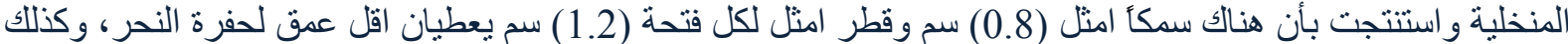
الجدار بسمك (0.8) سم و الفتحة بقطر (1.6) سم يعطيان اكبر طول لحفرة النحر ، وأن التجارب بدون استخدام جدار منخلي

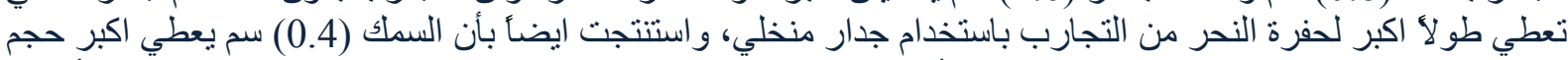

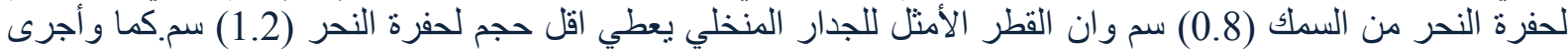

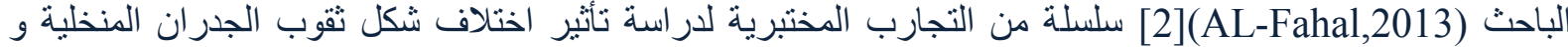

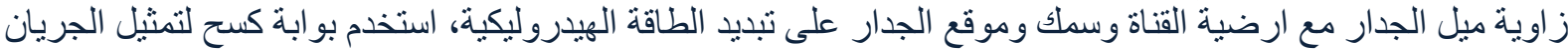

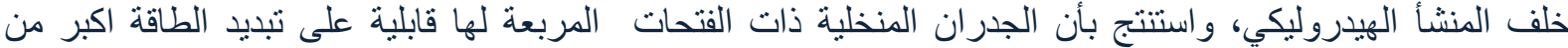
الجدران المنخلية ذات الفتحات الدائرية، وأن سمك الجدار ليس له مساهمة اضافية هامة في تبديد الطاقة.

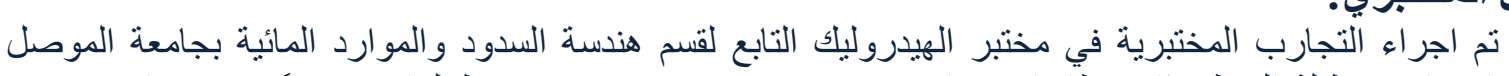

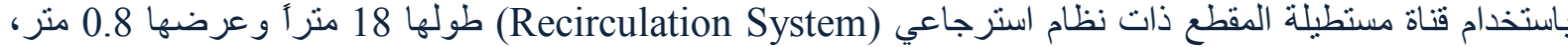

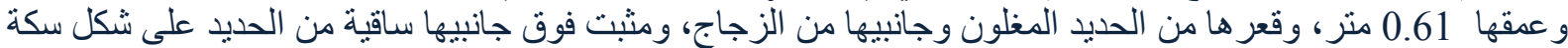

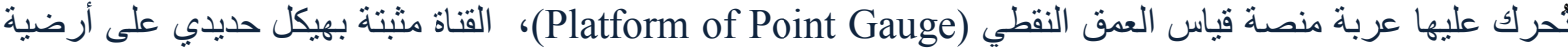

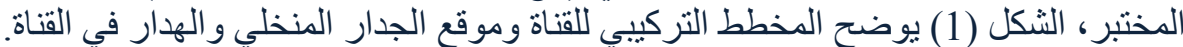

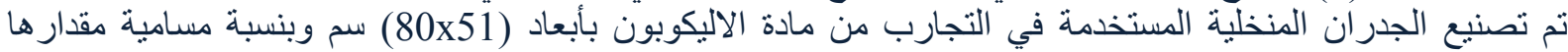

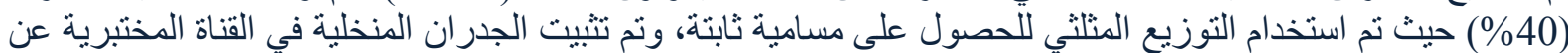

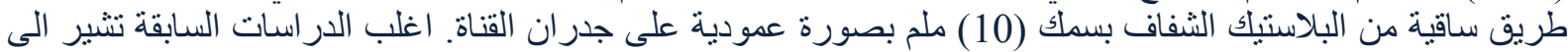

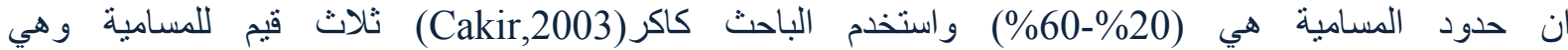

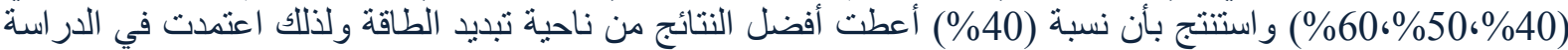
الحالية. تم استخدام نموذج من الحجر المكسر المار من منخل (9.525) ملم ومرتد على منخل (6.35) ملم بقطر (7.94)

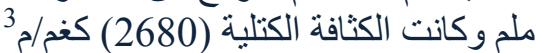


حياوي: دراسة مختبرية لتقليل النحر مؤخر الهارات باستخدام الجدران المنخلية

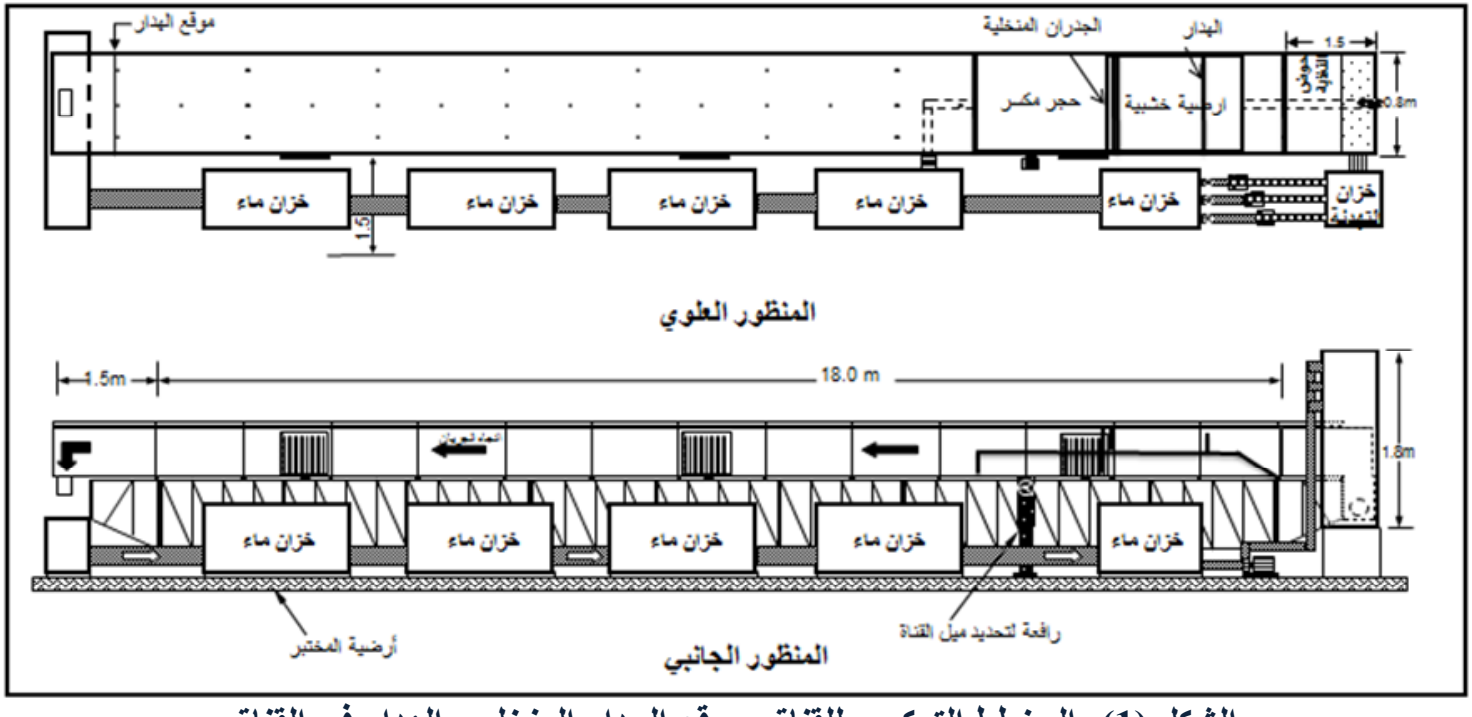

الثكل (1): المخطط التركيبي للقناة وموقع الجدار المنظلي والهدار في القناة

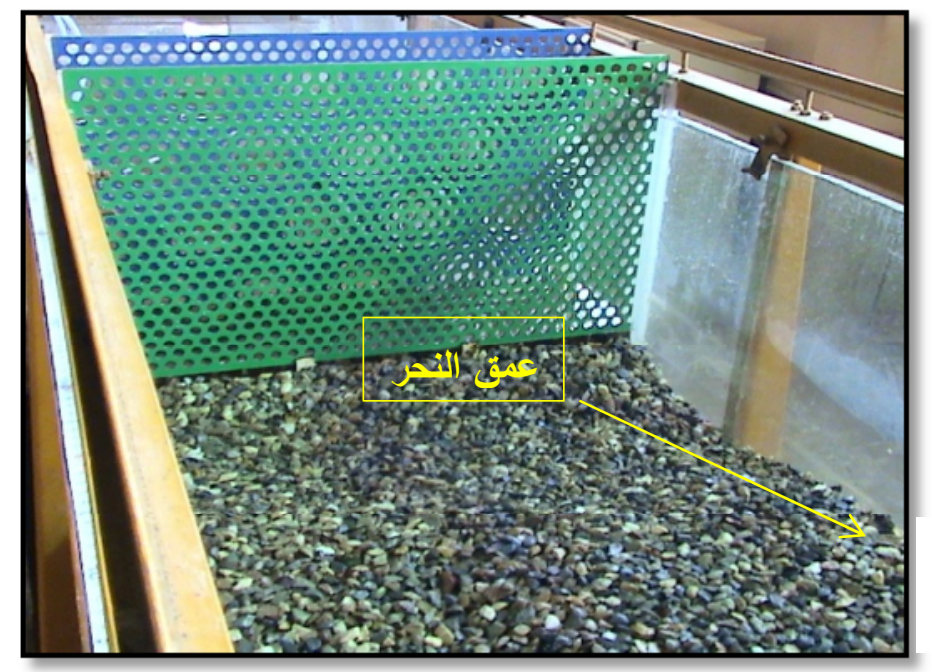

كما تم تثبيت الجدر ان المنخلية بصورة عمودية

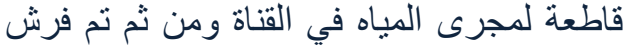

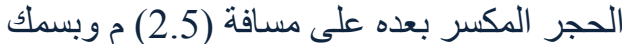

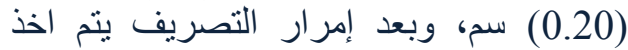
مناسيب الماء قبل وبعل وبعد الهدار و والجدران

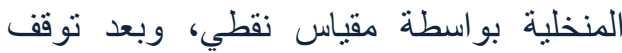
عملية النحر يتم إيقاف مرور التصريف ونيف ونؤخذ مناسيب طول و عمق حفرة النحر. و اللوحة رقم (1) توضح الجدران المنخلية الثنائية بقطر (1.6) سم و عمق وطول حفرة النحر.

لوحة رقم (1) : توضح الجدران المنظلية الثنائية بقطر (1.6)سم وعمق وطول حفرة النحر.

تحليل ومناقشئة النتائج: هنالك عدة عو امل مؤثرة على خصائل خصائص حفرة النحر مؤخر الجدران المنخلية ويمكن صباغة العلاقة الخاصـة بعمق النحر

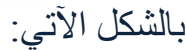

$D_{s}=f\left(q_{w}, \varnothing, S_{w}, D_{m}, g, \Delta \rho_{s}, P\right)$

كما يمكن صياغة العلاقة الخاصة بطول حفرة النحر بالثكل الآتي:-

$L_{s}=f\left(q_{w}, \varnothing, S_{w}, D_{m}, g, \Delta \rho_{s}, P\right)$

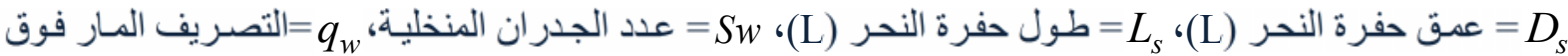
الهدار لوحدة عرض (L) (L)،

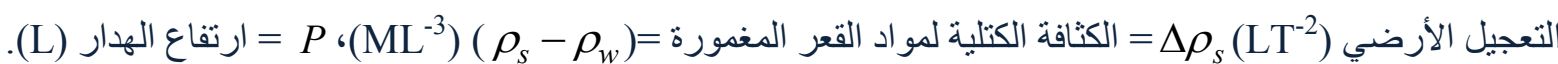
وباستخدام طريقة التحليل ألبعدي للمتغير ات في المعادلة (1) نم التوصل إلى العلاقة اللابعدية الآتية: 
$\frac{D_{s}}{P}=f\left(\frac{\emptyset}{P}, \frac{D_{m}}{P}, S w, F r\right)$

كما يمكن صياغة علاقة لا بعدية لطول حفرة النحر عن طريق التحليل البعدي بالثكل الاتي:

$\frac{L_{s}}{P}=f\left(\frac{\emptyset}{P}, \frac{D_{m}}{P}, S w, F r\right)$

Pن $=\frac{D_{S}}{P}$ المنخلي الى ارتفاع الهدار،

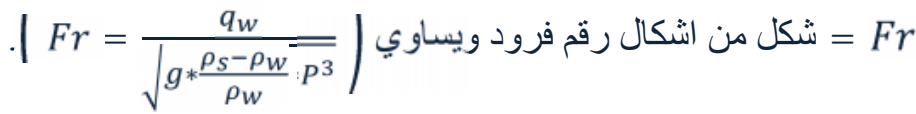

تأثير ارتفاع الهدار على العلاقة بين نسبة عمق النحر إلى معدل أقطار الحجر المكسر (Dm

(Frm)

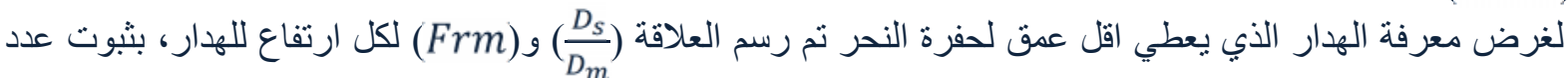
الجدران المنخلية وأقطار فتحات الجدار المنخلي ومعدل قطر مواد القعر ووجد بأن العلاقة الاسية هي افضل العلاقات بين) و (Frm) ويث تعطي اعلى معامل تحديد)

$\frac{D_{s}}{D_{m}}=a_{1} * \mathrm{Frm}^{b_{1}}$

= $=a_{1}, b_{1}$ Frm

و الجدول (1) يبين قيم المعاملات ومعامل التحديد للمعادلة أعلاه،

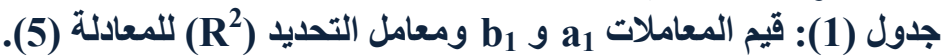

\begin{tabular}{|c|c|c|c|c|}
\hline Case & $\mathbf{R}^{2}$ & $\overline{a_{1}}$ & $\mathbf{b}_{1}$ & Figure No. \\
\hline $1-1.6-30$ & 0.9681 & 0.2781 & 0.9402 & \multirow{4}{*}{ 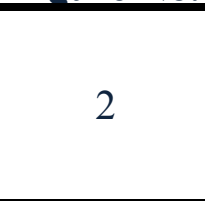 } \\
\hline $1-1.6-25$ & 0.9622 & 0.1818 & 1.0509 & \\
\hline 30-Classic & 0.9647 & 0.1141 & 1.1341 & \\
\hline 25-Classic & 0.8906 & 0.2866 & 1.0099 & \\
\hline $1-1.2-30$ & 0.9749 & 0.0763 & 1.2731 & \multirow{4}{*}{3} \\
\hline $1-1.2-25$ & 0.9906 & 0.0624 & 1.3132 & \\
\hline 30-Classic & 0.9647 & 0.1141 & 1.1341 & \\
\hline 25-Classic & 0.8906 & 0.2866 & 1.0099 & \\
\hline $1-0.8-30$ & 0.9726 & 0.0382 & 1.4425 & \multirow{4}{*}{4} \\
\hline $1-0.8-25$ & 0.9840 & 0.0146 & 1.701 & \\
\hline 30-Classic & 0.9647 & 0.1141 & 1.1341 & \\
\hline 25-Classic & 0.8906 & 0.2866 & 1.0099 & \\
\hline
\end{tabular}


حياوي: دراسة مختبرية لتقليل النحر مؤخر الهدارات باستخدام الجدران المنخلية

\begin{tabular}{|c|c|c|c|c|}
\hline $2-1.6-30$ & 0.9880 & 0.0588 & 1.3964 & \multirow{3}{*}{5} \\
\hline 2-1.6-25 & 0.9877 & 0.0416 & 1.4825 & \\
\hline 30-Classic & 0.9647 & 0.1141 & 1.1341 & \\
\hline 25-Classic & 0.8906 & 0.2866 & 1.0099 & \\
\hline $2-1.2-30$ & 0.9752 & 0.0448 & 1.4137 & \multirow{2}{*}{6} \\
\hline 2-1.2-25 & 0.9691 & 0.0238 & 1.5890 & \\
\hline 30-Classic & 0.9647 & 0.1141 & 1.1341 & \multirow{2}{*}{7} \\
\hline 25-Classic & 0.8906 & 0.2866 & 1.0099 & \\
\hline 2-0.8-30 & 0.9666 & 0.0040 & 2.0984 & \\
\hline 2-0.8-25 & 0.9561 & 0.0007 & 2.6237 & \\
\hline 30-Classic & 0.9647 & 0.1141 & 1.1341 & \\
\hline 25-Classic & 0.8906 & 0.2866 & 1.0099 & \\
\hline
\end{tabular}

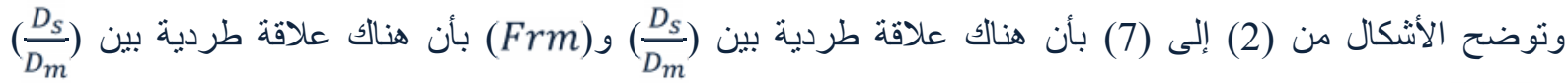

(Frm),

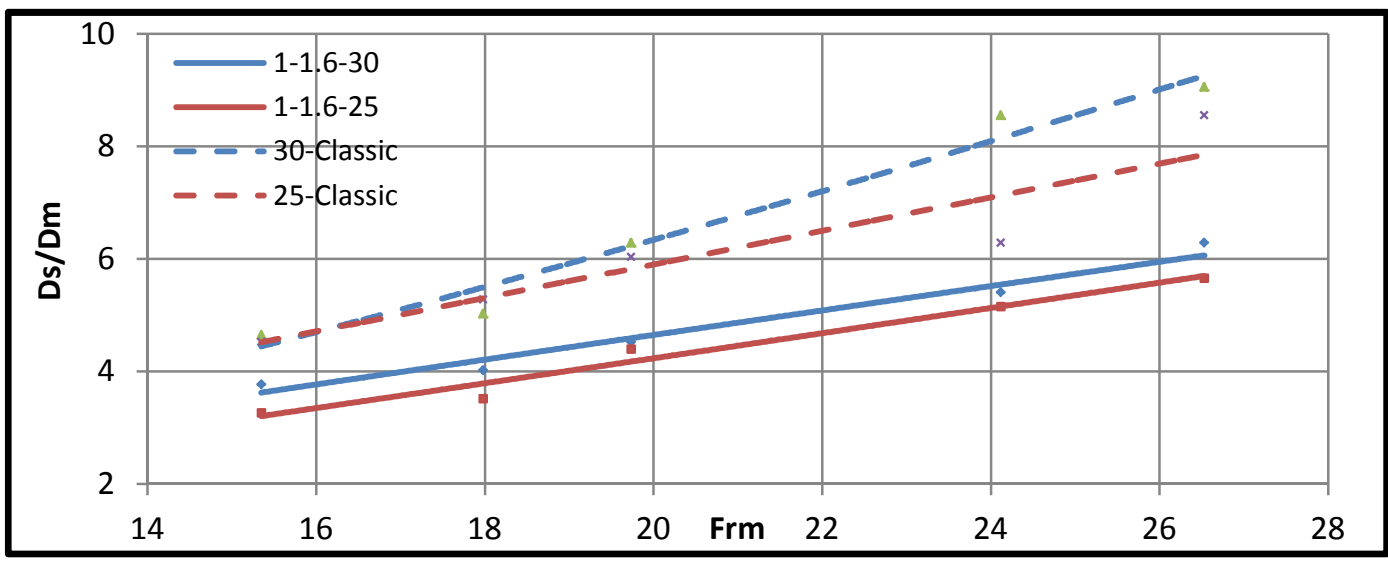

الثكل (2): العلاقة بين

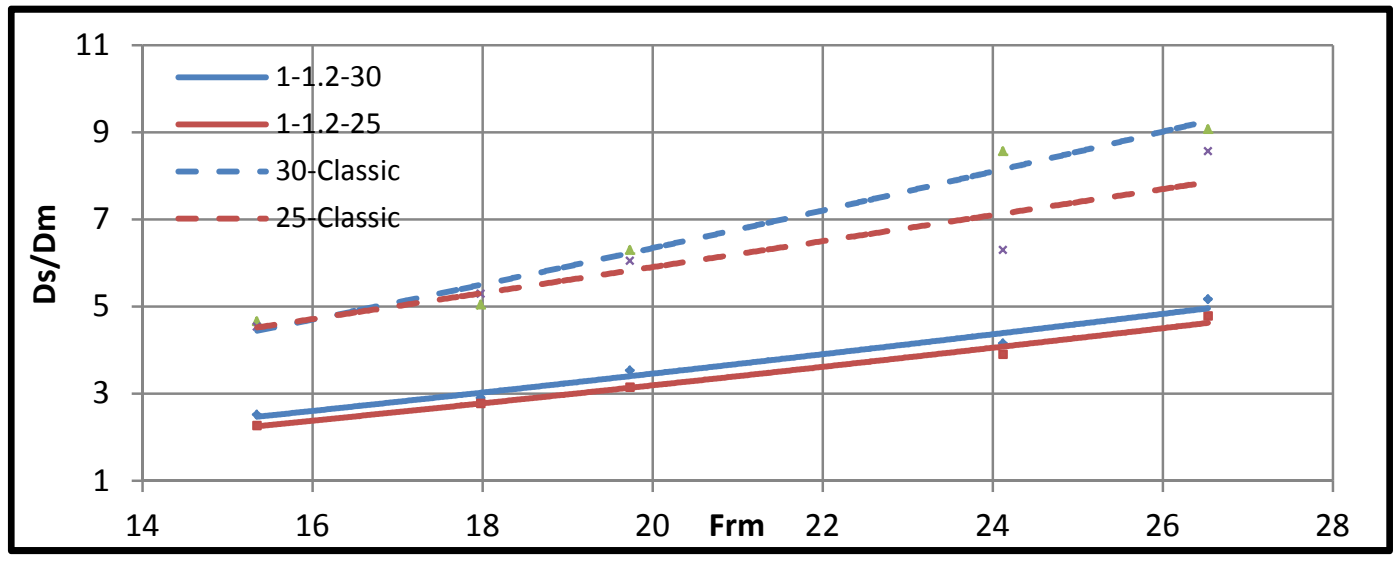

الثكل (3): العلاقة بين 


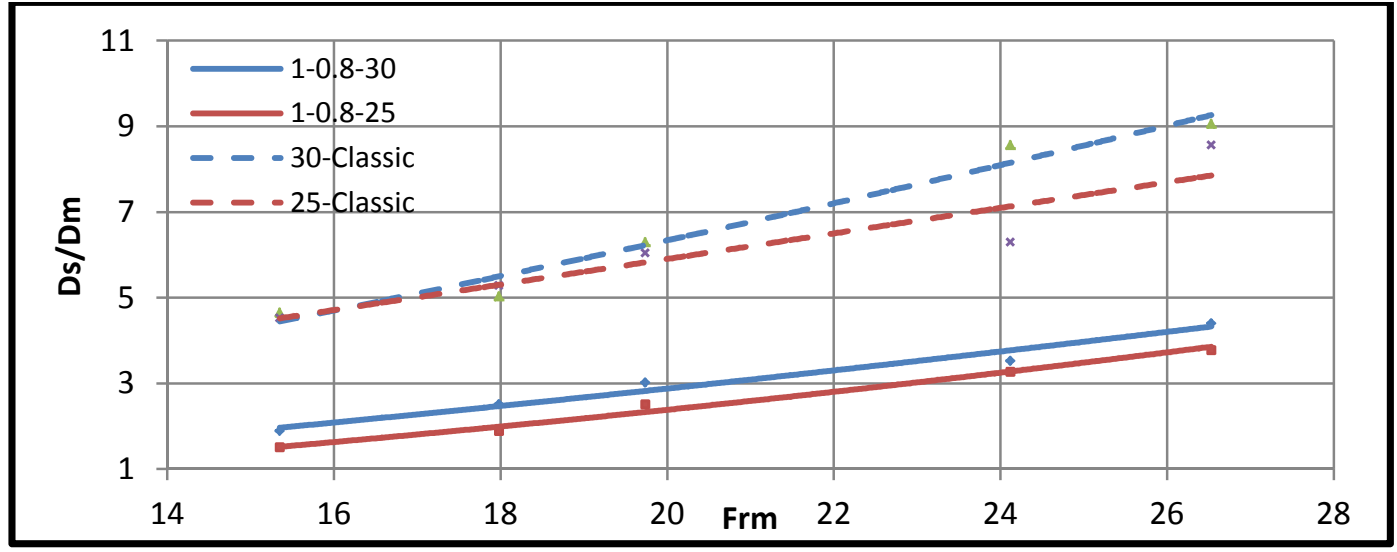

الثكل (4): العلاقة بين

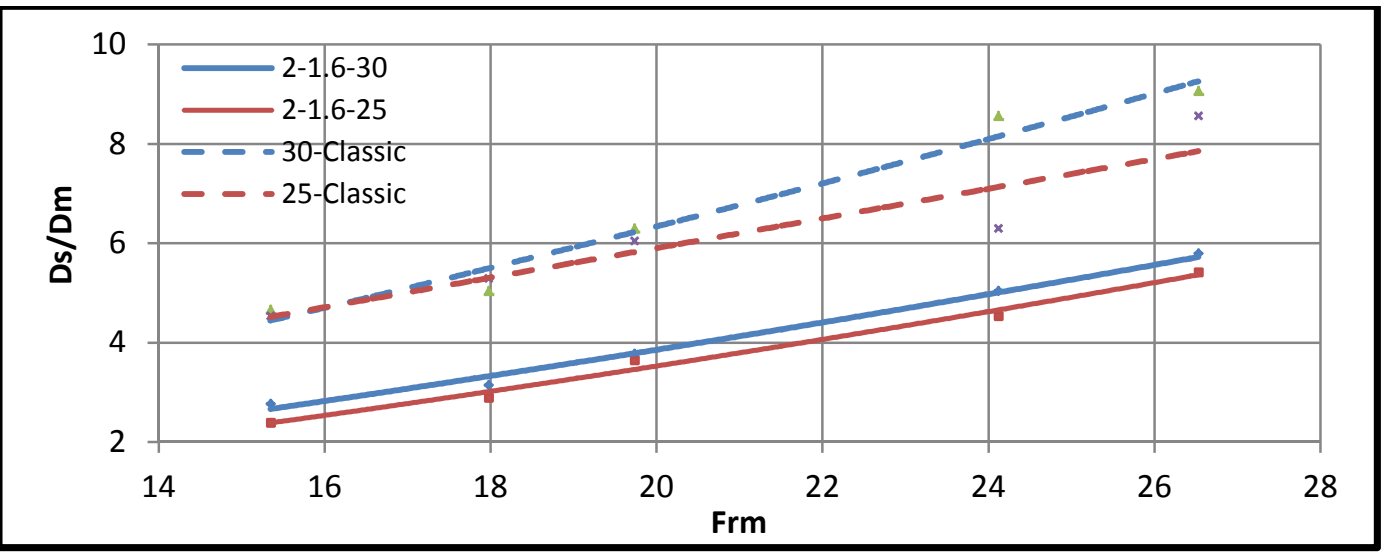

الشكل (5): العلاقة بين

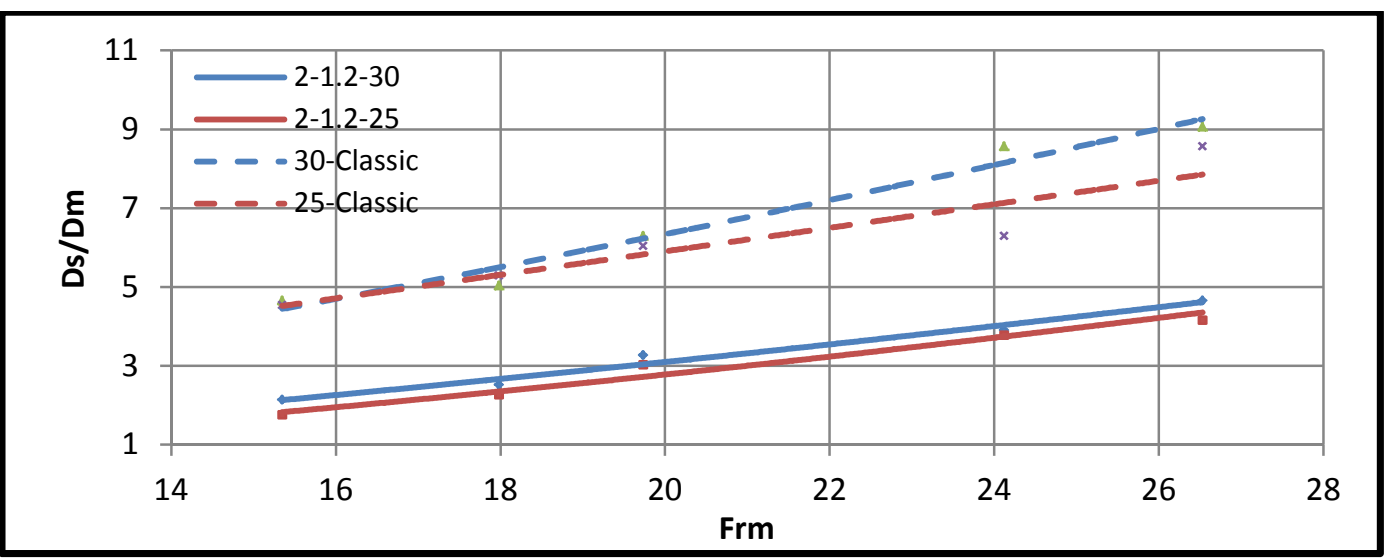

الشكل (6): العلاقة بين 


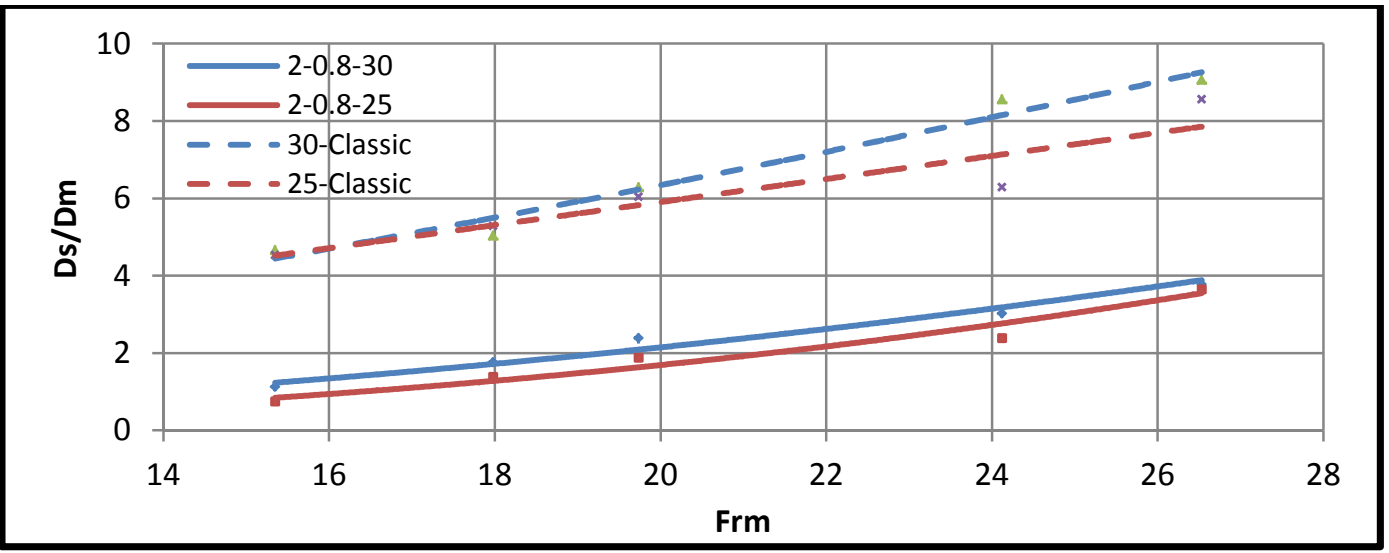

$$
\text { الثكل (7): العلاقة بين }
$$

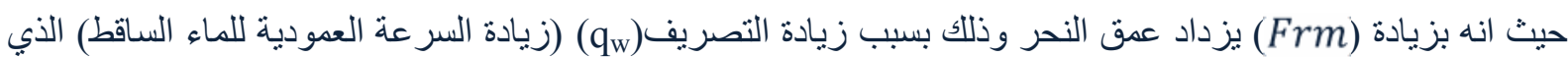

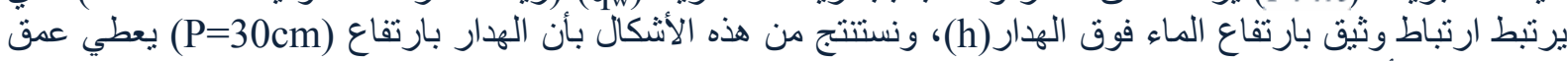

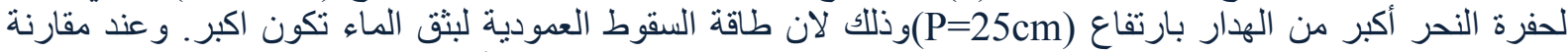

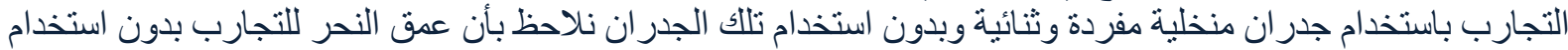

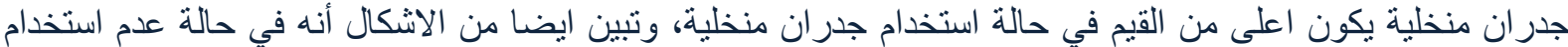

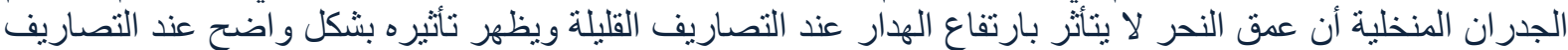

العالية.

تأثير ارتفاع الهدار على العلاقة بين نسبة طول حفرة النحر إلى معدل أقطار الحجر المكسر (

فرود (Frm)

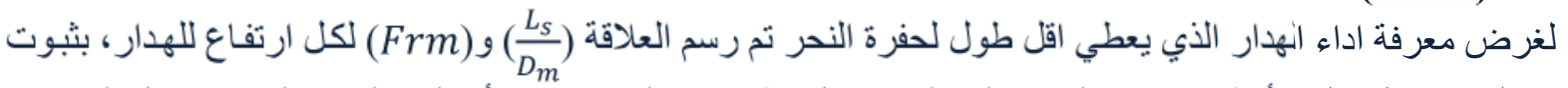
عدد الجدران المنخلية وأقطار فتحات الجدار المنظلي ومعدل قطر مواد القعر ووجد بأن العلاقة الاسية هي افضل العاء العلاقات بين

$\frac{L_{s}}{D_{m}}=a_{2} * \mathrm{Frm}^{b_{2}}$

. $=a_{2}, b_{2}$

و الجدول (2) يبين قيم المعاملات ومعامل التحديد للمعادلة اعلاه

جدول (2): قيم المعاملات a و b ومعامل التحديا (R2

\begin{tabular}{|c|c|c|c|c|}
\hline Case & $\mathbf{R}^{2}$ & $\mathbf{a}_{2}$ & $b_{2}$ & Figure No. \\
\hline $1-1.6-30$ & 0.9729 & 1.0536 & 1.3116 & \multirow{4}{*}{ 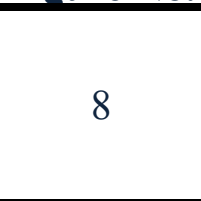 } \\
\hline $1-1.6-25$ & 0.9863 & 0.7936 & 1.3723 & \\
\hline 30-Classic & 0.9152 & 12.997 & 0.7188 & \\
\hline 25-Classic & 0.9803 & 15.063 & 0.6403 & \\
\hline $1-1.2-30$ & 0.9813 & 0.5382 & 1.4561 & \multirow{3}{*}{9} \\
\hline $1-1.2-25$ & 0.9812 & 0.7448 & 1.2991 & \\
\hline 30-Classic & 0.9152 & 12.997 & 0.7188 & \\
\hline
\end{tabular}


No. 5

Dec. 2014

\begin{tabular}{|c|c|c|c|c|}
\hline 25-Classic & 0.9803 & 15.063 & 0.6403 & \\
\hline $1-0.8-30$ & 0.9526 & 0.7361 & 1.2963 & \multirow{4}{*}{10} \\
\hline $1-0.8-25$ & 0.9733 & 0.5685 & 1.3184 & \\
\hline 30-Classic & 0.9152 & 12.997 & 0.7188 & \\
\hline 25-Classic & 0.9803 & 15.063 & 0.6403 & \\
\hline $2-1.6-30$ & 0.9896 & 0.9423 & 1.3178 & \multirow{4}{*}{11} \\
\hline $2-1.6-25$ & 0.9887 & 0.8908 & 1.3027 & \\
\hline 30-Classic & 0.9152 & 12.997 & 0.7188 & \\
\hline 25-Classic & 0.9803 & 15.063 & 0.6403 & \\
\hline $2-1.2-30$ & 0.9821 & 0.4412 & 1.4801 & \multirow{4}{*}{12} \\
\hline $2-1.2-25$ & 0.9617 & 0.2267 & 1.6504 & \\
\hline 30-Classic & 0.9152 & 12.997 & 0.7188 & \\
\hline 25-Classic & 0.9803 & 15.063 & 0.6403 & \\
\hline $2-0.8-30$ & 0.9645 & 0.1510 & 1.7478 & \multirow{4}{*}{13} \\
\hline $2-0.8-25$ & 0.9640 & 0.0624 & 1.9771 & \\
\hline 30-Classic & 0.9152 & 12.997 & 0.7188 & \\
\hline 25-Classic & 0.9803 & 15.063 & 0.6403 & \\
\hline
\end{tabular}

وتوضح الأشكال من (8) إلى (13) بأن هناك علاقة طردية بين $\left(\frac{L^{2}}{D_{m}}\right)$ و)

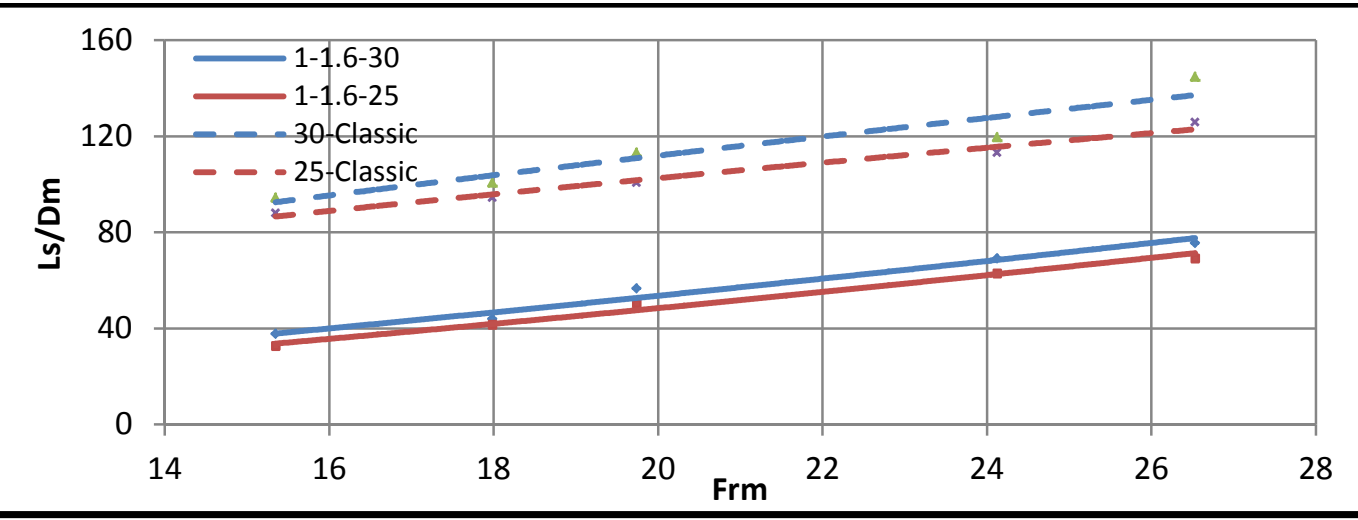

الثكل (8): العلاقة بين

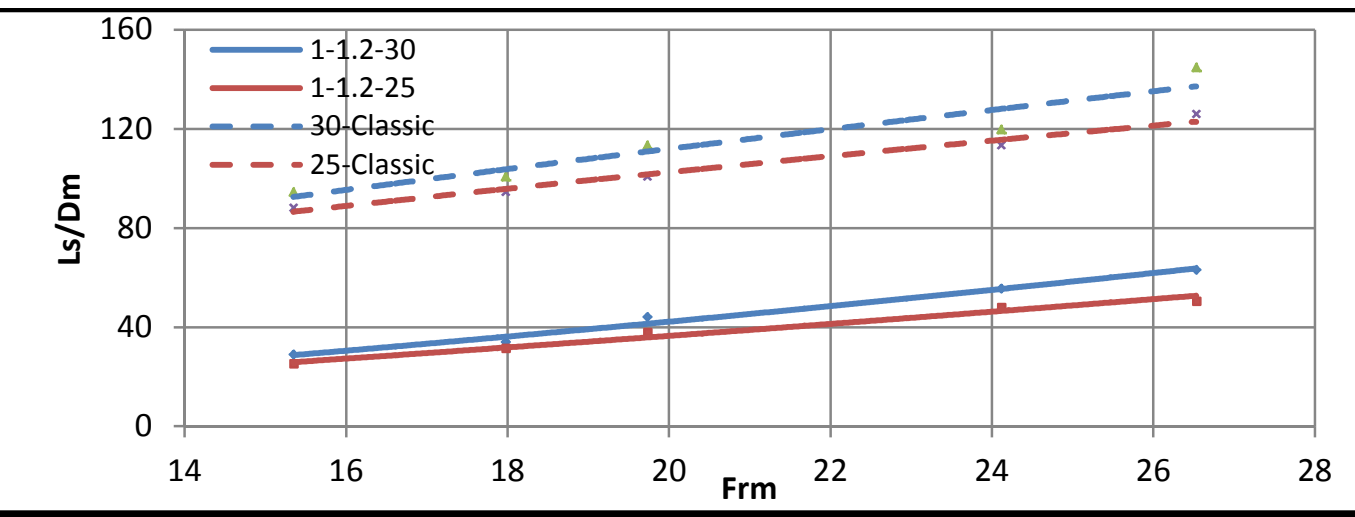

الشكل (9): العلاقة بين 
حياوي: دراسة مختبرية لتقليل النحر مؤخر الهدارات باستخدام الجدران المنخلية

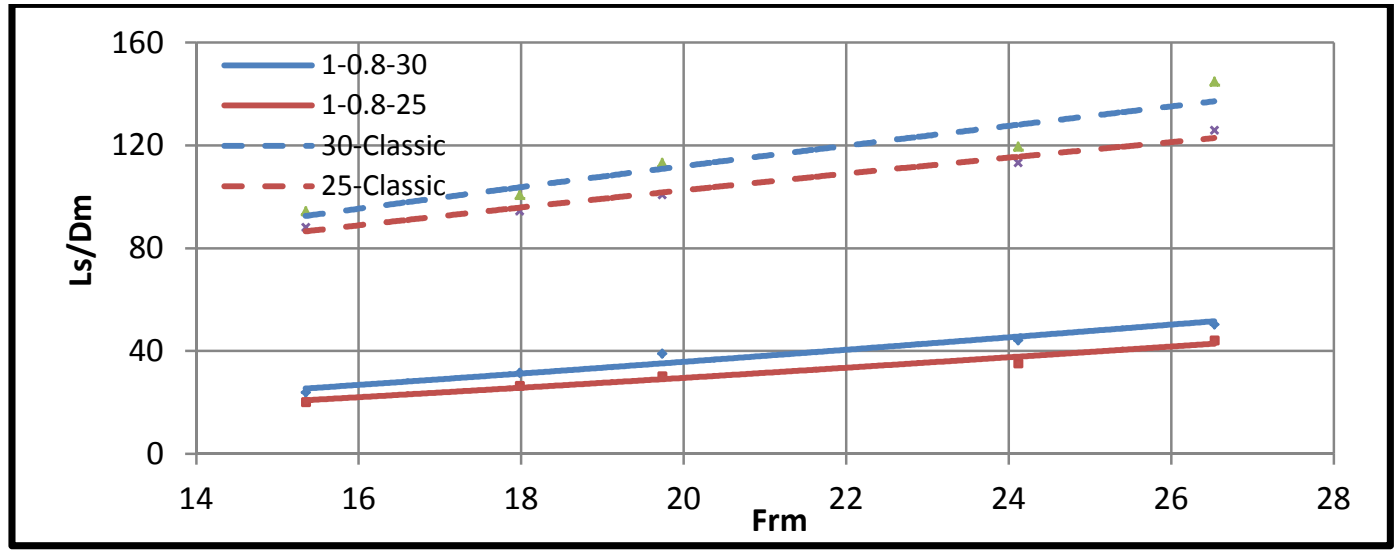

الشكل (10): العلاقة بين

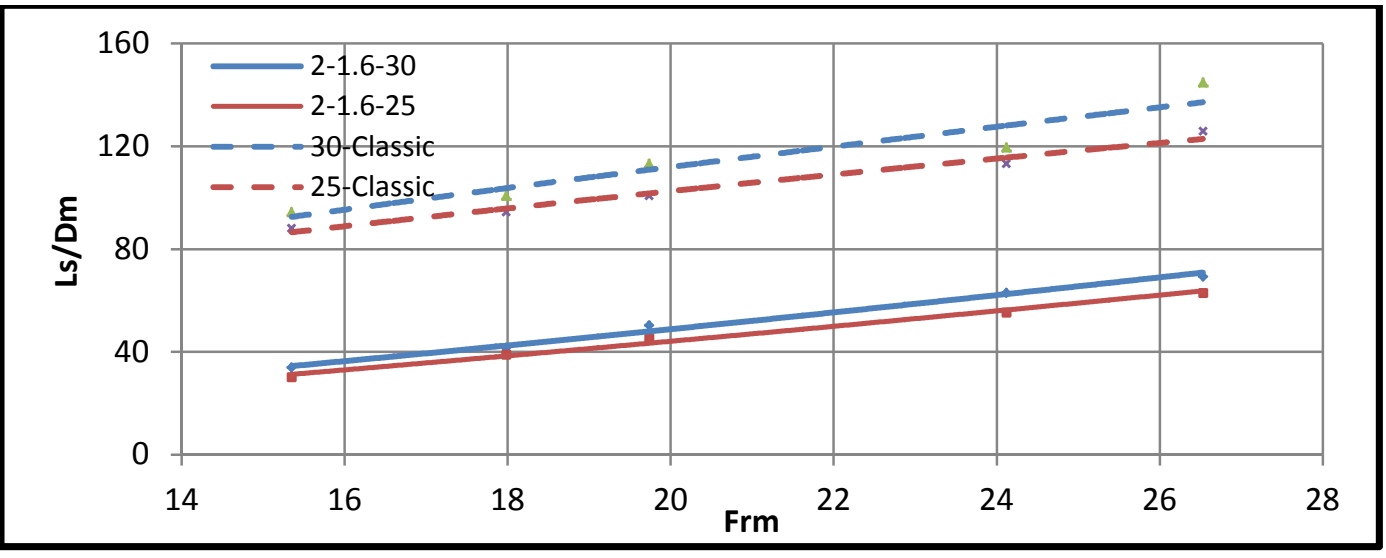

الشكل (11): العلاقة بين

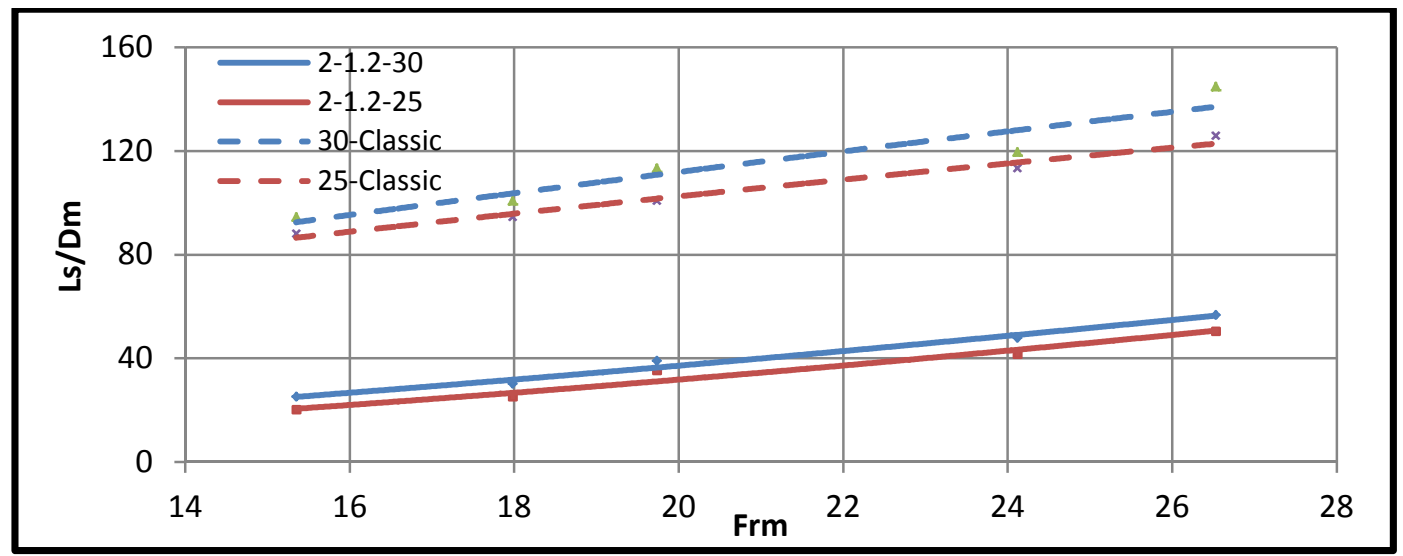

الشكل (12): العلاقة بين 


\section{$\begin{array}{llll}\text { Al-Rafidain Engineering } & \text { Vol.22 } & \text { No. } 5 & \text { Dec. } 2014\end{array}$}

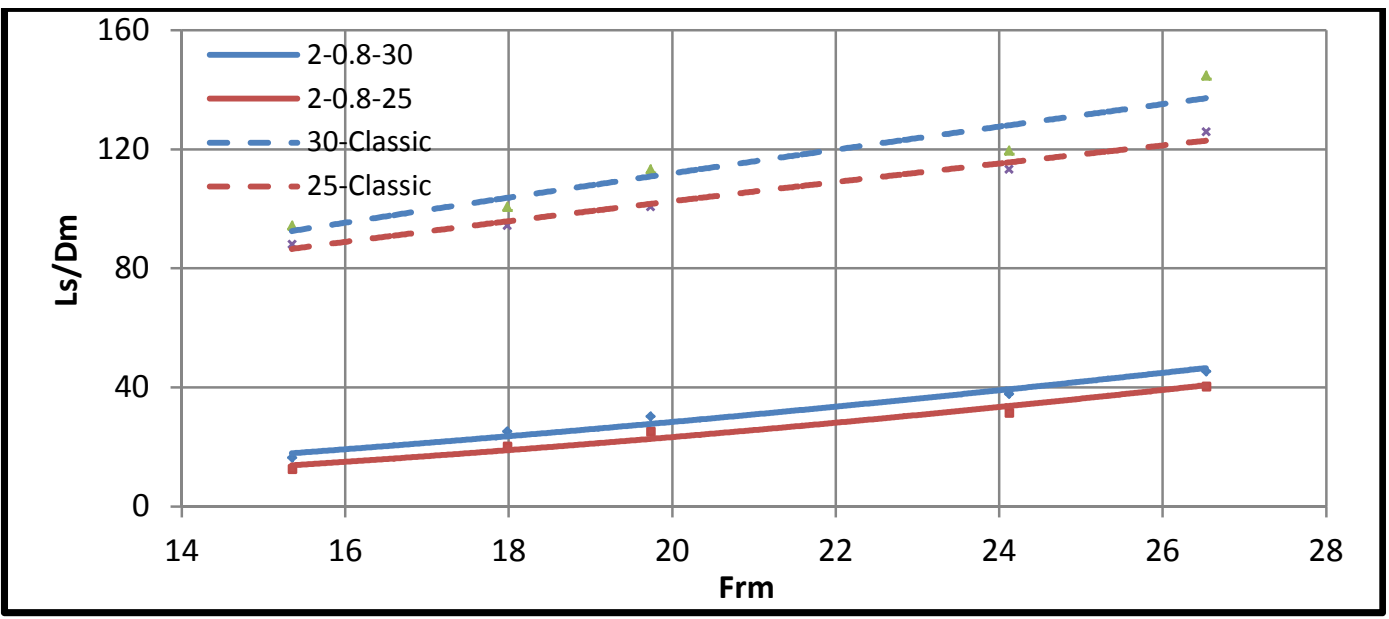

$$
\text { الثكل (13): العلاقة بين }
$$

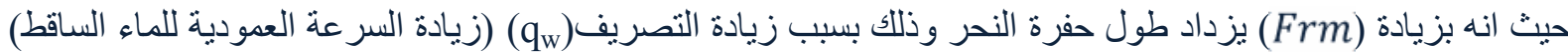

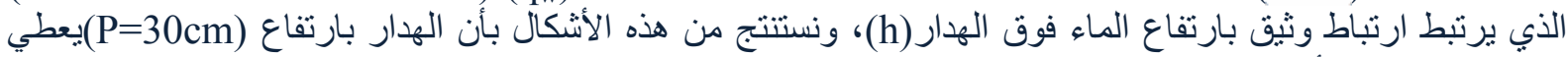

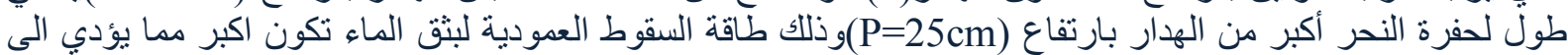

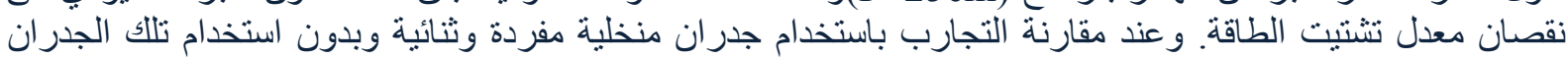

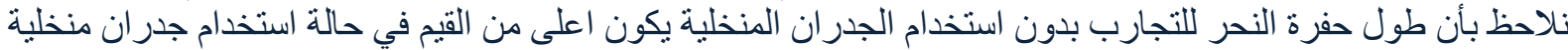
بنسبة (28-83.33)\% بالنسبة لعمق النحر وبنسبة (47.37-85.71)\% بالنسبة لطول حفرة النحر.

\section{مخطط سطح الماء:}

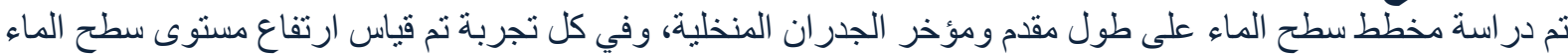

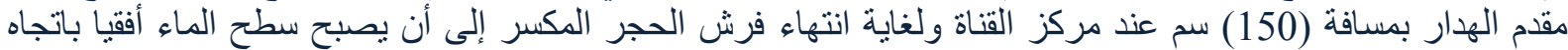

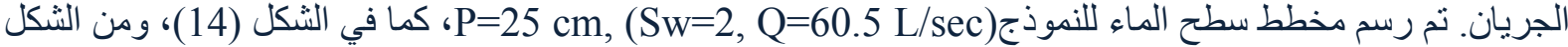

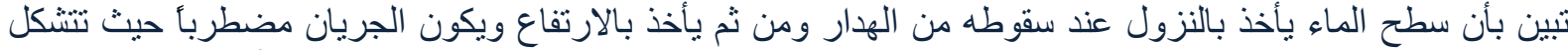

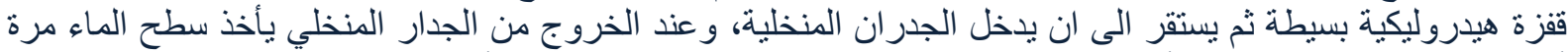

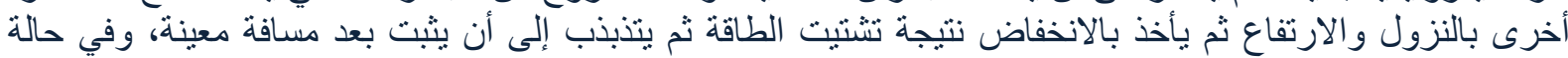
استخدام جدران منخلية ثنائية يلاحظ تكون قفزة هيدروليكية بين الجدارين.

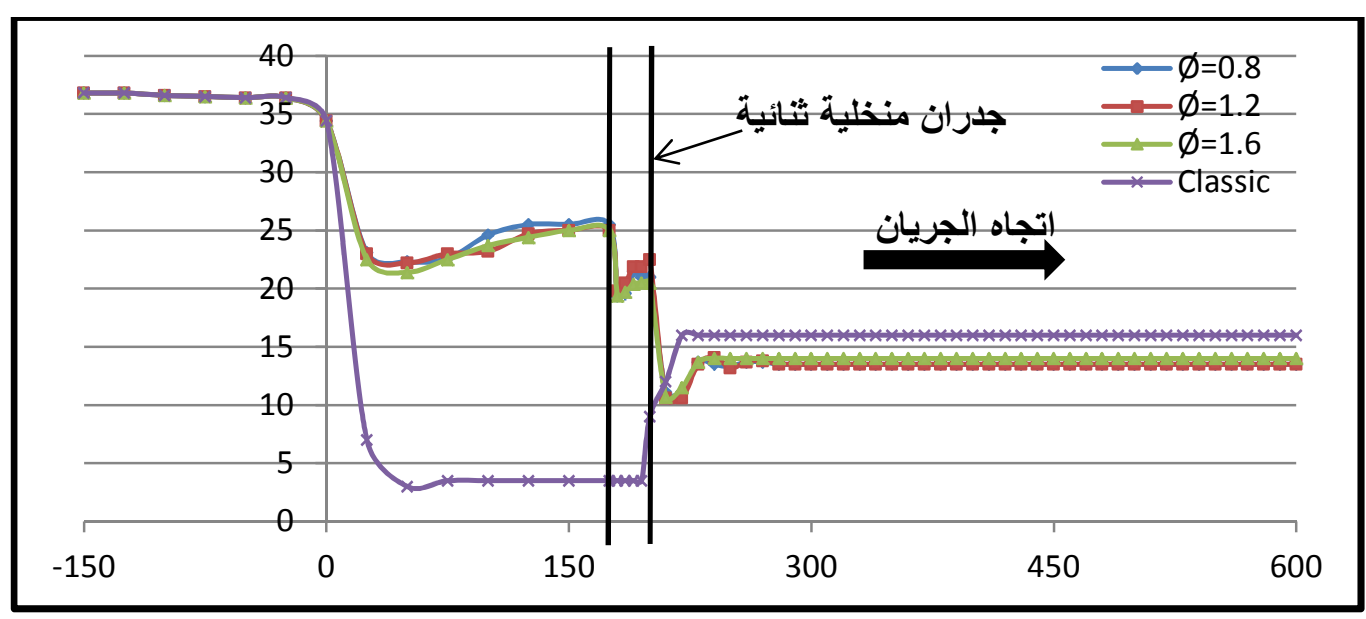

شكل (14): شكل سطح الماء عندما (P=25 cm , Sw=2 , Q=60.5 L/sec) 
علاقة وضعية لحساب نسبة عمق النحر الى ارتفاع الهدار(

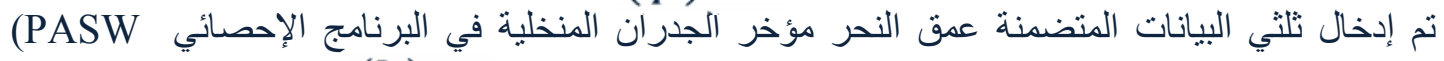
Statistics 18)

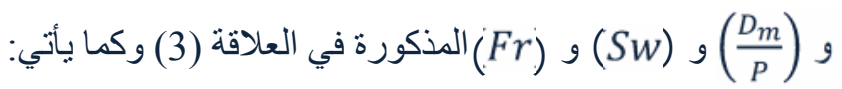

$\frac{D s}{P}=0.006 * F r^{1.37} *\left(\frac{D m}{P}\right)^{-2.318} *\left(\frac{\varnothing}{P}\right)^{0.734} * S w^{-0.202}$

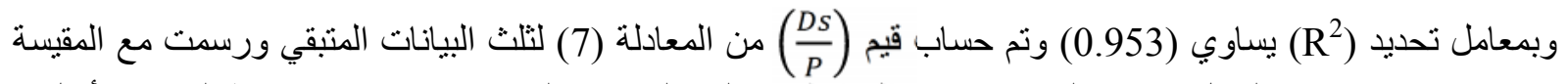
مختبريا وكما موضح بالثكل (15) و الذي ييين مدى الترابط بين القيم المقيسة والمحسوبة ونقاربها مع خط التو افق الأمثل.

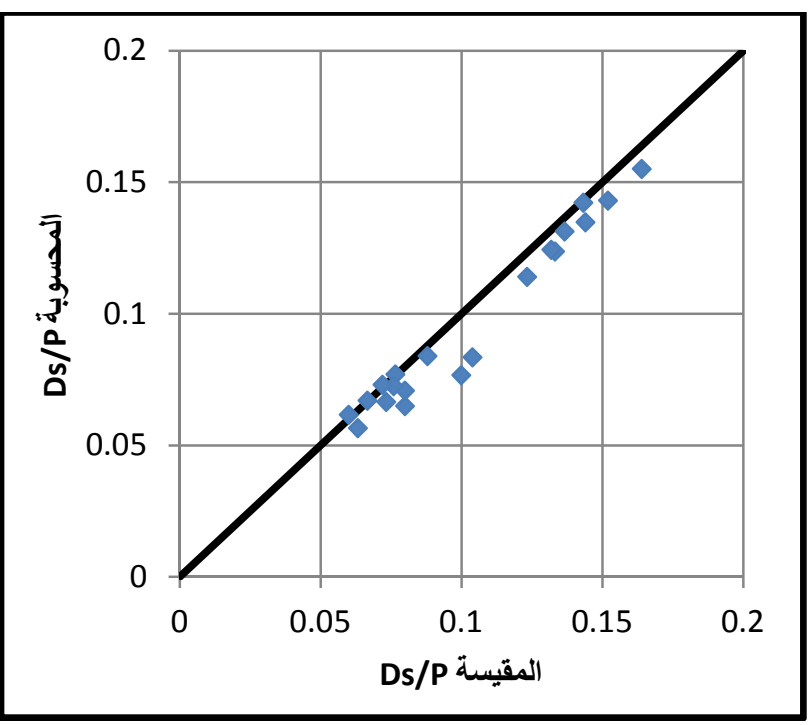

شكل (15): مقارنة نسبة عمق النحر الى ارتفاع الهدار(Ps) المحسوب من المعادلة(7) والمقيسة مختبرياً

\section{علاقة وضعية لحساب نسبة طول حفرة النحر الى ارتفاع الهدار(}

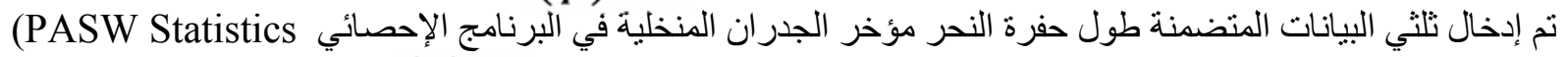

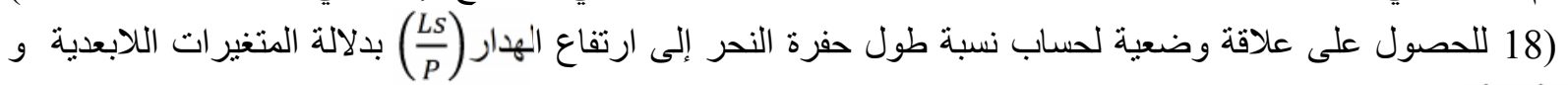

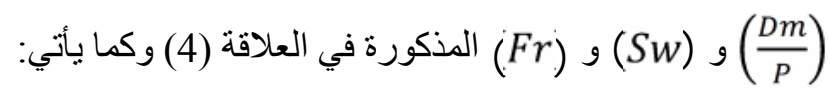

$\frac{L s}{P}=0.041 * F r^{1.358} *\left(\frac{D m}{P}\right)^{-2.477} *\left(\frac{\varnothing}{P}\right)^{0.723} * S w^{-0.164}$

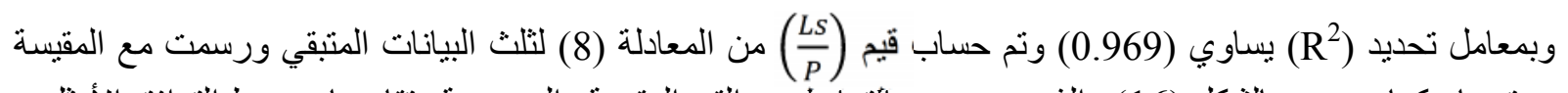

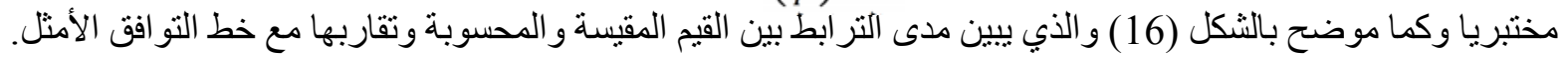




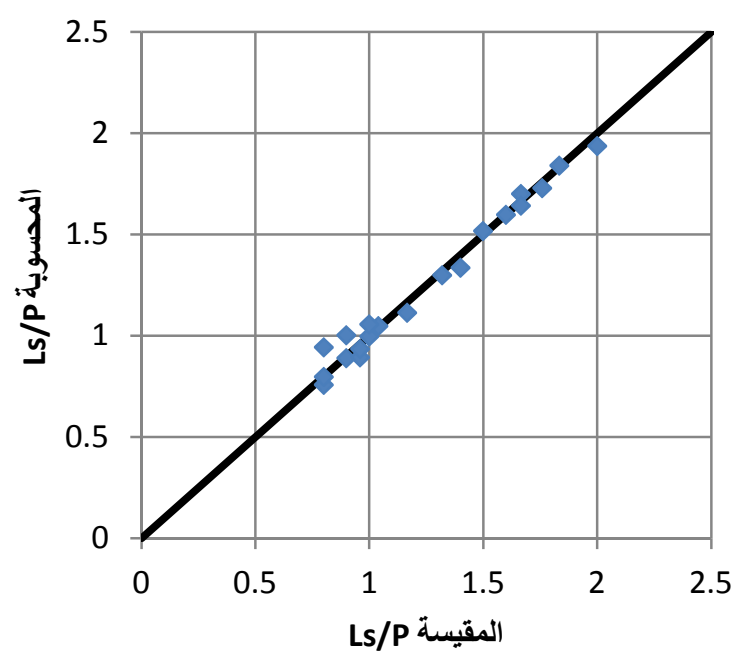

شكل (16): مقارنة نسبة طول النحر الى ارتفاع الهدار(Ps) المحسوب من المعادلة(8) والمقيسة مختبرياً

Nash-)

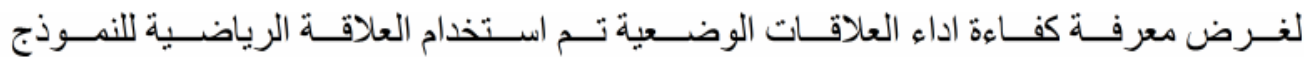
:[6](Sutcliffe model efficiency coefficient - 1970

$E=1-\frac{\sum\left(O_{o}-O_{m}\right)^{2}}{\sum\left(O_{o}-\overline{O_{o}}\right)^{2}}$

E

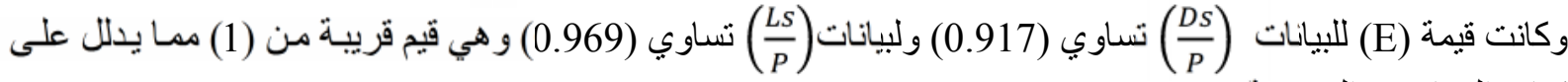

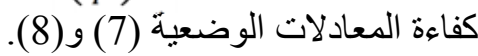
| (الاستنتاجات:

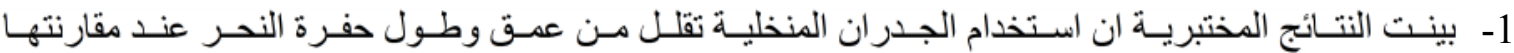

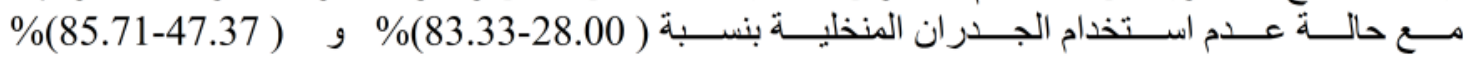

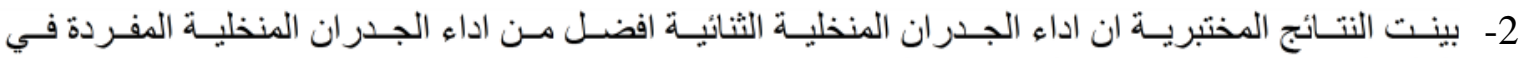

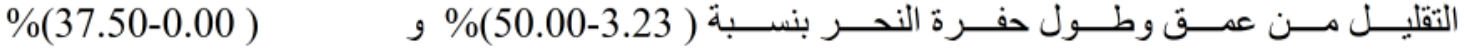

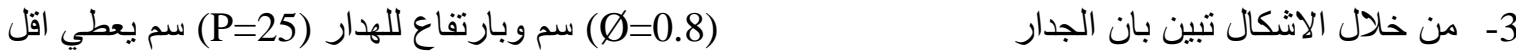
4- تم استتباط علاقتين وضعيتين لحساب نسبة عمق وطول حفرة النحر لارتفاع الهدار (2011). "در اسة مختبرية للنحر خلف الجدران المنخلية المستخدمة في تبديد المبل

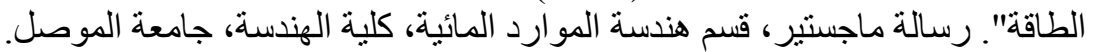

2. Al-Fahal, A.S.M. (2013), " Laboratory Study of Hydraulic Energy Dissipation Using Screen With Circular \& Square Holes", M.Sc. thesis, Department of Civil Engineering, College of Engineering University of Tikrit.

3. Balkis, G., (2004), "Experimental Investigation of Energy Dissipation through Inclined 
Screens", M.Sc. thesis, Department of Civil Engineering, Middle East Technical University, Ankara, Turkey.

4. Cakir, P.(2003), "Experimental Investigation of Energy Dissipation through Screen " , M.Sc. thesis, Department of Civil Engineering, Middle East Technical University, Ankara, Turkey.

5. Mahmoud, H.A. (2010), "Laboratory Study of Energy Dissipation Using Screen Walls ", M.Sc. thesis, Department of Civil Engineering, College of Engineering University of Dohok.

6. Nash, J. E. and Sutcliffe, J. V.(1970): River flow forecasting through conceptual models, Part I - A discussion of principles, J. Hydrol., 10, 282-290. 